\title{
Journal of Lung Diseases \& Treatment
}

\section{Idiopathic Lymphoid Interstitial Pneumonia: A Case Report}

Herath HMLY', Punyajeewa UAG ${ }^{1}$, Gurunayake B ${ }^{2}$, Upul BKMA ${ }^{1}$, Abeykoon AJMP' and Siribaddana AD ${ }^{1 *}$

${ }^{1}$ Respiratory Medicine Unit 1, Teaching Hospital Kandy, Sri Lanka

${ }^{2}$ Department of Radiology, Teaching Hospital Anuradhapura, Sri Lanka

\begin{abstract}
Idiopathic Lymphoid Interstitial Pneumonia (LIP) is considered to be a rare interstitial lung disease. The initial American Thoracic Society/European Respiratory Society (ATS/ERS) International multidisciplinary consensus paper on Idiopathic Interstitial Pneumonia of 2002, LIP was classified as one of the seven major idiopathic interstitial lung diseases. Since the first description of LIP in 1969, so far only 14 biopsy proven cases of idiopathic LIP have been described in the literature. The clinical course is reported to be highly variable in this rare form of interstitial pneumonia.
\end{abstract}

Keywords: Idiopathic lymphoid; Interstitial pneumonia; Loci; Dyspnoea

\section{Introduction}

The incidence and prevalence of disease of LIP is unknown. In a prospective study Cha et al., found only 3 patients with idiopathic LIP out of a total of 2,989 subjects ( 1,167 surgical lung biopsies) over a period of 15 years [1-4]. The 2013 revision of the ATS/ERS international multidisciplinary consensus classification LIP was reclassified as a rare interstitial lung disease [5]. This statement also emphasized the need of excluding all known secondary causes and pulmonary lymphoma which warrants extensive investigation before arriving at a diagnosis of idiopathic disease. LIP is known to be associated with several diseases and conditions including Sjögrens syndrome, HIV and Epstein-barr virus infections, Autoimmune hemolytic anemia, Myasthenia gravis, Pernicious anemia, Hashimoto's thyroiditis, Chronic active hepatitis and Primary biliary cirrhosis [6]. The diagnosis of idiopathic lymphoid interstitial pneumonia can only be made when all possible causes are excluded.

Sjögrens syndrome is one of the most common syndromes associated with LIP. Recent observations have indicated that both $\mathrm{B}$ and $\mathrm{T}$ cells play an essential role in the immune response in the Sjögrens syndrome [7]. Both genetic and epigenetic mutations are involved in the B-cell associated development of Sjögrens syndrome as evidenced by occurrence of aberrant DNA methylation in B cells at the genetic at-risk loci [8]. Understanding the levels of epigenetic modifiers in LIP patients derived B cells might be a novel direction for the study of initiation and development of this rare human lung disorder.

We report a 74 years old male in whom radiological findings were compatible with idiopathic LIP and the diagnosis confirmed histopathologically.

\section{Case Report}

74 years old male diagnosed was referred to specialized respiratory diseases unit with worsening dyspnoea. His NYHA (New York Heart Association) dyspnoea score worsened from grade I to grade IV during a period of three months. He did not have cough or constitutional symptoms such as night sweats or loss of weight. He gave a history of 3 pack years of smoking, stopped 20 years ago. He did not have clinical features suggestive of connective tissue diseases, chronic liver cell diseases or any hematological diseases. There was no history of known exposures to organic or inorganic dusts.

On examination he was dyspnoeic at rest with a $\mathrm{SPO}_{2}$ of $82 \%$ on air. He had grade II finger clubbing. There was no plethora, lymphadenopathy, inflamed or deformed joints or skin manifestations of connective tissue disorders. His respiratory rate was 22 breaths per minute and auscultation revealed bilateral basal fine end inspiratory crepitations.

His full blood count, renal, liver and thyroid function tests were normal. Hepatitis B, HIV and EBV screening was negative. Plasma immunoglobulin fraction was normal and plasma autoantibody screening was negative for rheumatoid factor, anti-cyclic citrullinated peptide and a panel of anti nuclear antibodies. The Erythrocyte Sedimentation Rate was $68 \mathrm{~mm} / 1^{\text {st }} \mathrm{h}$ and $\mathrm{C}$ reactive protein level was $6 \mathrm{IU}(0-6)$.

Spirometry revealed moderate restriction with a forced vital capacity (FVC) of $1.92 \mathrm{~L}$ which was $65 \%$ of predicted value and a FEV1/ FVC ratio of $98 \%$ (FEV1=Forced Expiratory Volume in 01 second).

His chest radiograph showed bilateral reticular and cystic opacities with basal predominance. The High-resolution CT scan of the lungs showed extensive areas of ground-glass attenuation with thin-walled cysts in all three zones. Few perivascular cysts were also noted. Lung bases showed evidence of septal thickening. However, bilateral subpleural honeycombing was evident even though it is considered to be rare in LIP. Consolidations and nodular opacities were absent (Figures 1-4).

An open lung biopsy was carried out and the histology showed heavy infiltrate of lymphocytes admixed with plasma cells and neutrophils expanding the interstitium in the peri-bronchiolar and inter-alveolar regions. There were lymphoid follicles with active germinal center formation seen focally (Figure 5).

The diagnosis of idiopathic lymphocytic interstitial pneumonia was made as all possible secondary causes were excluded during the work up.

The patient was treated initially with prednisolone $0.5 \mathrm{mg} / \mathrm{kg}$ for one year along with prophylaxis for osteoporosis and Pneumocystis pneumonia. The patient showed remarkable improvement, his NYHA dyspnoea score improved from grade 4 to grade 2 within four weeks of steroid therapy. The subsequent lung function test showed significant improvement with recording a FVC of $82 \%$ of predicted.

${ }^{*}$ Corresponding author: Siribaddana AD, Respiratory Medicine Unit 1, Teaching Hospital Kandy, Sri Lanka, Tel: 94773684814; E-mail: chamath1122@gmail.com

Received January 17, 2017; Accepted January 23, 2017; Published January 27, 2017

Citation: Herath HMLY, Punyajeewa UAG, Gurunayake B, Upul BKMA, Abeykoon AJMP, et al. (2017) Idiopathic Lymphoid Interstitial Pneumonia: A Case Report. Lung Dis Treat 3: 118. doi:10.4172/2472-1018.1000118

Copyright: (c) 2017 Herath HMLY, et al. This is an open-access article distributed under the terms of the Creative Commons Attribution License, which permits unrestricted use, distribution, and reproduction in any medium, provided the original author and source are credited. 
Citation: Herath HMLY, Punyajeewa UAG, Gurunayake B, Upul BKMA, Abeykoon AJMP, et al. (2017) Idiopathic Lymphoid Interstitial Pneumonia: A Case Report. Lung Dis Treat 3: 118. doi:10.4172/2472-1018.1000118

Page 2 of 3

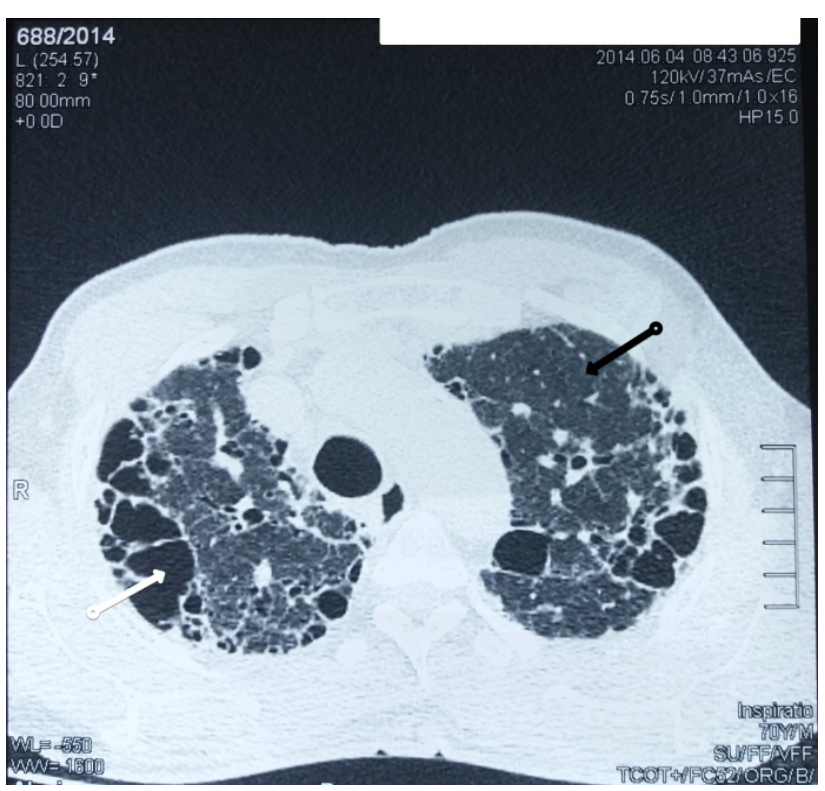

Figure 1: HRCTs at diagnosis.

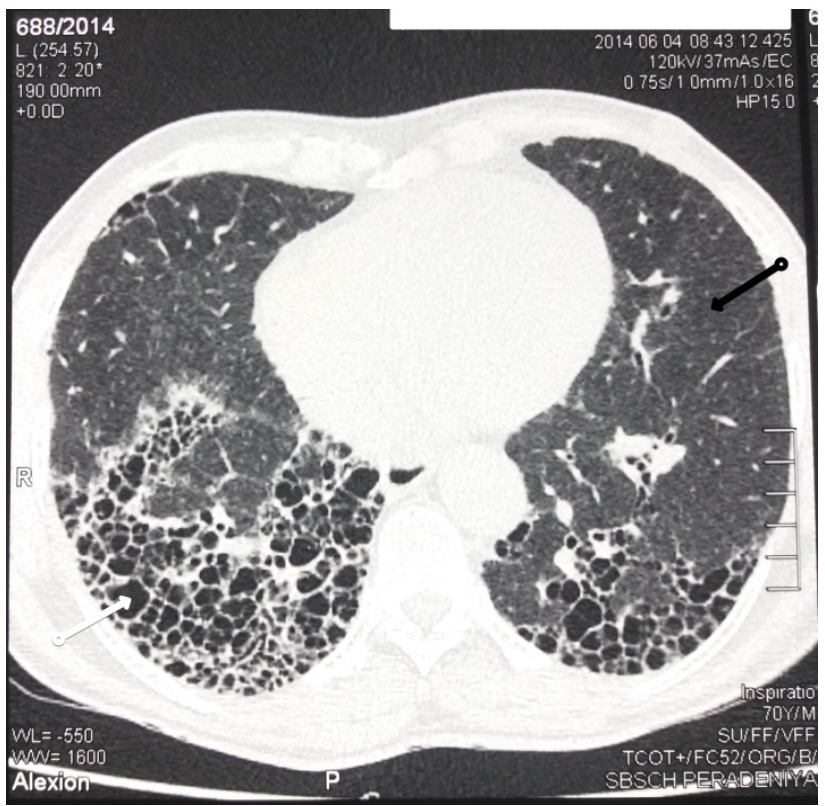

Figure 2: HRCTs at diagnosis.

After one year of treatment, HRCT remained static. Attempted reduction of steroid dose caused symptomatic relapse with a drop in FVC. Since the patient was steroid dependent, he was commenced on steroid sparing treatment. Azathioprine was commenced at a dose and steroids were gradually tailed off up to a dose of $5 \mathrm{mg}$ a day. He was kept under close observation for development of known secondary causes and lymphoma. The patient remained well having a NYHA dyspnea score of 2 for 18 months after the diagnosis until he died of a stroke.

\section{Discussion}

LIP appears to occur between 40-56 years of age and most of the patients are females. Our patient was a male who presented with the disease in the $8^{\text {th }}$ decade. Although our patient presented with gradual onset of dyspnoea, he did not have constitutional symptoms such as fever, night sweats and weight loss which are described in the literature [6].

Two chest radiographic patterns for lymphocytic interstitial pneumonia have been described: basilar with an alveolar component and diffuse with associated honeycombing. Our patient showed basilar predominance with linear and cystic opacities with diffuse alveolar opacification. The High-resolution CT scan of the lungs showed extensive areas of ground-glass attenuation with thin-walled cysts in all three zones extensively. The dominant CT finding described in literature are ground glass opacification with thin walled cysts, lung nodules, thickening of the broncho-vascular bundles, interlobular

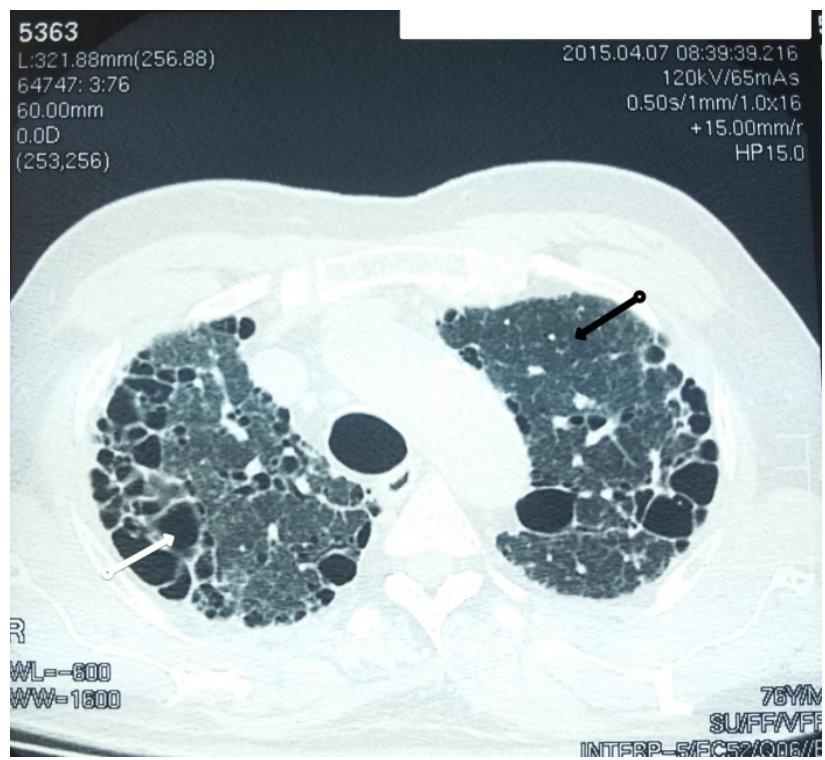

Figure 3: Follow up HRCTs done after one year of treatment. Black arrowsground glass opacities, White arrows-cysts.

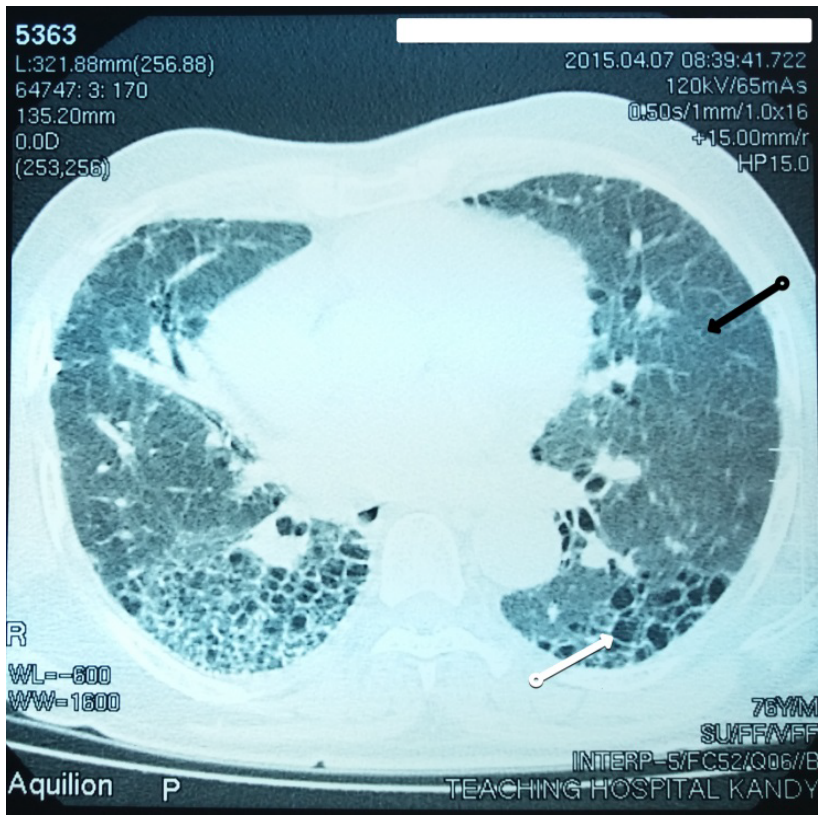

Figure 4: Follow up HRCTs done after one year of treatment. Black arrowsground glass opacities, White arrows-cysts. 


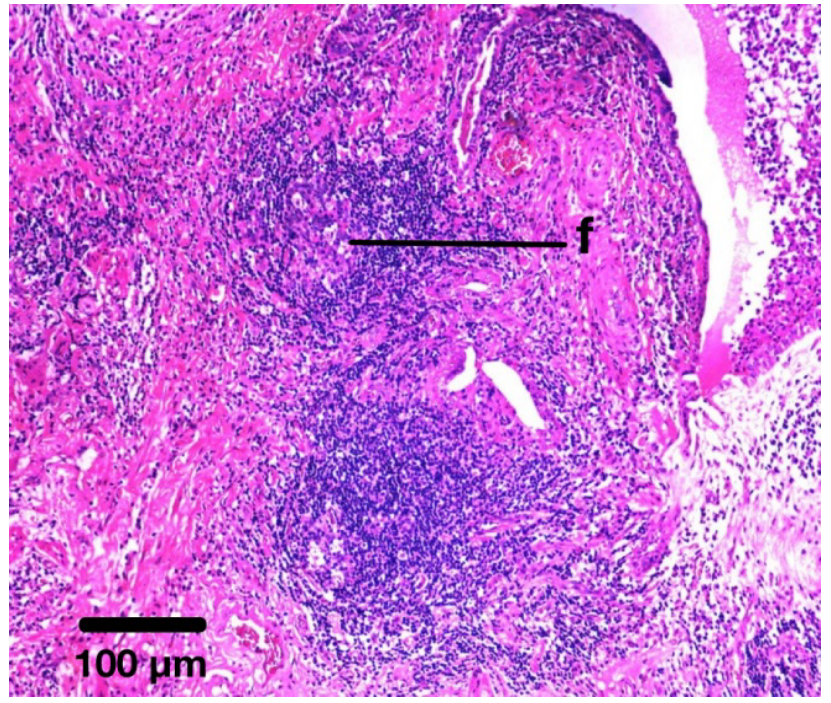

Figure 5: Lymphoid follicles (f) with active germinal center formation in the interstitium $\mathrm{H}$ and $\mathrm{E}$ 100X.

septal thickening and widespread consolidations. Our patient showed extensive cyst formation with ground glass opacification. Septal thickening was noted in the bases. But, there were no nodules and extensive areas of consolidation were not seen. Bilateral sub-pleural honeycombing was evident even though it is considered to be rare in LIP $[6,9,10]$.

Open lung biopsy of our patient showed heavy infiltrate of lymphocytes admixed with plasma cells expanding the interstitium in the peri-bronchiolar and inter-alveolar regions. There were lymphoid follicles with active germinal center formation seen focally. These findings confirm with the international literature. Granuloma formation, intra-alveolar organization or macrophage accumulation which have been described in the literature, was not seen in our patient $[11,12]$.

Treatment regimens for LIP have not been well established in the absence of controlled treatment data due to the rareness of the disorder. According to the available limited number of case records, LIP specially the secondary form, appears to respond to steroid therapy which to date is considered as the mainstay of treatment. Our patient responded well to steroids showing significant improvement in the dyspnoea score and FVC. His condition was steroid dependent. Therefore, he had to be given azathioprine with a maintenance dose of $5 \mathrm{mg}$ of prednisolone daily, to which he responded well $[4,9,12]$.

His HRCT was repeated after one year which showed no progression of the disease. The amount of cysts and the extent of ground glass opacifications remained static.

\section{Acknowledgements}

Prof. N. Ratnatunga, Department of Pathology, Faculty of Medicine, University of Peradeniya, Sri Lanka. Lanka.

Staff of Department of Respiratory Medicine, Teaching Hospital Kandy, Sri

\section{References}

1. ATS (2002) American Thoracic Society/European Respiratory Society International Multidisciplinary Consensus Classification of the Idiopathic Interstitial Pneumonias. Am J Resp Crit Care Med 165: 277-304.

2. Leibow AA, Carrington CB (1969) The interstitial pneumonias. Frontiers of Pulmonary Radiology, Grune \& Stratton, NY, USA 1: 102-141.

3. Teruya-Feldstein J, Temeck BK, Sloas MM (1995) Pulmonary malignant lymphoma of mucosa-associated lymphoid tissue (MALT) arising in a pediatric HIV positive patient. Am J Surg Pathol 19: 357-363.

4. Cha SI, Fessler MB, Cool CD, Schwarz MI, Brown KK (2006) Lymphoid interstitial pneumonia: clinical features, associations and prognosis. Eur Respir J 28: 364-369.

5. ATS (2013) American Thoracic Society/European Respiratory Society international multidisciplinary consensus classification of the idiopathic interstitial pneumonias. Am J Crit Care Med 188: 733-748

6. Kokosi MA, Nicholson AG, Hansell DM, Wells AU (2016) Rare idiopathic interstitial pneumonias: LIP and PPFE and rare histologic patterns of interstitial pneumonias: AFOP and BPIP. Respirology, 21: 600-614.

7. Martel C (2014) Pulmonary manifestations of Sjögren's syndrome. Rev Med Interne 35: 524-530.

8. Miceli-Richard C (2016) Overlap between differentially methylated DNA regions in blood B lymphocytes and genetic at-risk loci in primary Sjögren's syndrome. Ann Rheum Dis 75: 933-940.

9. Johkoh T, Müller NL, Pickford HA, Hartman TE, Ichikado K, et al. (1999) Lymphocytic interstitial pneumonia: thin-section CT findings in 22 patients. Radiology 212: 567-572.

10. Mang CM, Claudia G, Katharina S, Leopold S, Alexander AB (2007) What Every Radiologist Should Know About Idiopathic Interstitial Pneumonias. Radio Graphics 27: 595-615.

11. Nicholson AG (2001) Lymphocytic interstitial pneumonia and other lymphoproliferative disorders in the lung. Semin Respir Crit Care Med 22: 409-422.

12. Koss MN, Hochholzer L, Langloss JM, Wehunt WD, Lazarus AA (1987) Lymphoid interstitial pneumonia: clinicopathological and immunopathological findings in 18 cases. Pathology 19: 178-185. 